\title{
Dioecious Silene latifolia plants show sexual dimorphism in the vegetative stage
}

\author{
Jitka Zluvova*, Jiri Zak, Bohuslav Janousek, Boris Vyskot
}

\begin{abstract}
Background: Prior to this study, no differences in gene expression between male and female dioecious plants in the vegetative state had been detected. Among dioecious plants displaying sexual dimorphism, Silene latifolia is one of the most studied species. Although many sexually dimorphic traits have been described in S. latifolia, all of them are quantitative, and they usually become apparent only after the initiation of flowering.

Results: We present RT-PCR-based evidence that in S. latifolia, sexual dimorphism in gene expression is present long before the initiation of flowering. We describe three ESTs that show sex-specific (two male specific and one female specific) transcription at the rosette stage before the first flowering season.

Conclusions: To our knowledge, this study provides the first molecular evidence of early pre-flowering sexual dimorphism in angiosperms.
\end{abstract}

\section{Background}

Sexual dimorphism (the systematic difference in form or other trait(s) not present in sexual organs between individuals of different sex in the same species) is a widely studied phenomenon in animal models [1] and in humans [2]. Much less is known about sexual dimorphism in vascular dioecious plants (reviewed in [3]).

Among vascular plants displaying sexual dimorphism, Silene latifolia is (together with Fragaria virginiana [4]) the most studied species. The first study on sexually dimorphic traits in S. latifolia was performed already in the 19th century [5], and since that time many sexually dimorphic traits have been described (e. g., [6-9]). However, the only known genes involved in sexual dimorphism are those involved in the control of flower development. During flower development, sexual dimorphism starts to occur very early. At the morphological level, the central zone of the floral meristem is significantly smaller in males than in females [10]. This is caused by cell division arrest in male tissues [11]. The difference between male and female flower bud morphology is preceded by differences at the gene expression level. Developmental pathways involved in the

\footnotetext{
* Correspondence: jitka@ibp.cz

Department of Plant Developmental Genetics, Institute of Biophysics AS CR v. v. i., Kralovopolska 135, 61265 Brno, Czech Republic
}

switch of male or female flower program have been also identified [12,13].

The differential expression of some genes probably results from different modes of selection operating in males and females: males are limited in their reproductive success by access to mates, whereas females are more limited by resources [14]. In animals, the evolution of the sexual dimorphism is primarily driven by competition between males and selection for traits recognized by females as marks of male fitness (reviewed in [15]). Similar principles are probably also at work in animal pollinated plants. In S. latifolia, odor-compounds involved in pollinator attraction differ significantly between sexes, suggesting that selection for higher attractiveness among competing males is mediated by the sensory ecology of the pollinator [16]. In addition, males produce on average up to 16 times more flowers than pollinated females [17]. This difference in flower number is probably driven by a combination of male competition, and, at least partly, by a higher consumption of resources by developing seeds in pollinated female flowers, which probably results in a trade off between seed size and flower number. The difference in flower number is, indeed, less pronounced in nonpollinated females, which produce on average 4 times fewer flowers than males $[17,18]$. Yet, selection for increased flower number in males is hypothesized to be 
the primary mechanism for the further evolution of sexual dimorphism in other traits [8]. It also seems reasonable to expect that differences in the vegetative parts of plants evolved in concert with different flowers types or architecture of inflorescences carried by the plant [3]. Dawson and Geber [3] pointed out that many sexually dimorphic traits could evolve as a consequence of their correlation with other sexually dimorphic traits and so they need not be of adaptive value. Correlations between flower size and the size of the stem leaves have been reported by several independent studies (reviewed by [3]). Steven et al. [18] suggested that variation in sexlimited genes with pleiotropic effects and/or linkage between sex limited loci occurs in S. latifolia. They statistically predicted that selection for increased flower numbers in males along with weak selection for increased flower size in females could lead to dimorphic evolution in several other traits including leaf mass [18].

Almost all of the sexually dimorphic traits in S. latifolia described so far become apparent only after the initiation of flowering. Notable exceptions to this pattern include: sex-dimorphism in the long-term survival of buried seeds and burial induced dormancy in S. latifolia [19], sex-dimorphism in emergence time [20] and in the time to flowering $[20,21]$. We present the first molecular evidence that sexually dimorphic gene expression is present in S. latifolia even at the rosette stage, a long time before the initiation of flowering, and describe three ESTs with sex-specific gene expression.

\section{Results and discussion}

We re-tested the expression patterns of 22 available S. latifolia ESTs previously described according to Northern blots or Virtual Northerns [22-24] as preferentially expressed in male flowers and/or early stamen (for the list of the ESTs chosen for this study, see Additional file 1: Supplementary table S1). Fewer genes than previously claimed have expression limited to male flower buds suggesting the importance of RT-PCR analyses in this case. Only six out of 15 genes previously described as male flower bud specific were expressed in male flower buds only and not in the leaves or in female flower buds (Figure 1A). Two genes, originally described as male flower bud specific, were expressed in male flower buds earlier than in female flower buds (Figure 1B). Twelve genes were expressed in all samples tested (Figure 1C). For the comparison of the previously published data and our results see Additional file 2: Supplementary table S2. We also found one EST (Men-470) expressed exclusively in male flower buds and leaves and one EST (CCLS79.1) expressed exclusively in female flower buds and leaves (Figure 1D). We also serendipitously found one new $550 \mathrm{bp}$ long EST as a "by-product" of PCR amplification of Men-262 (Figure 1D) and
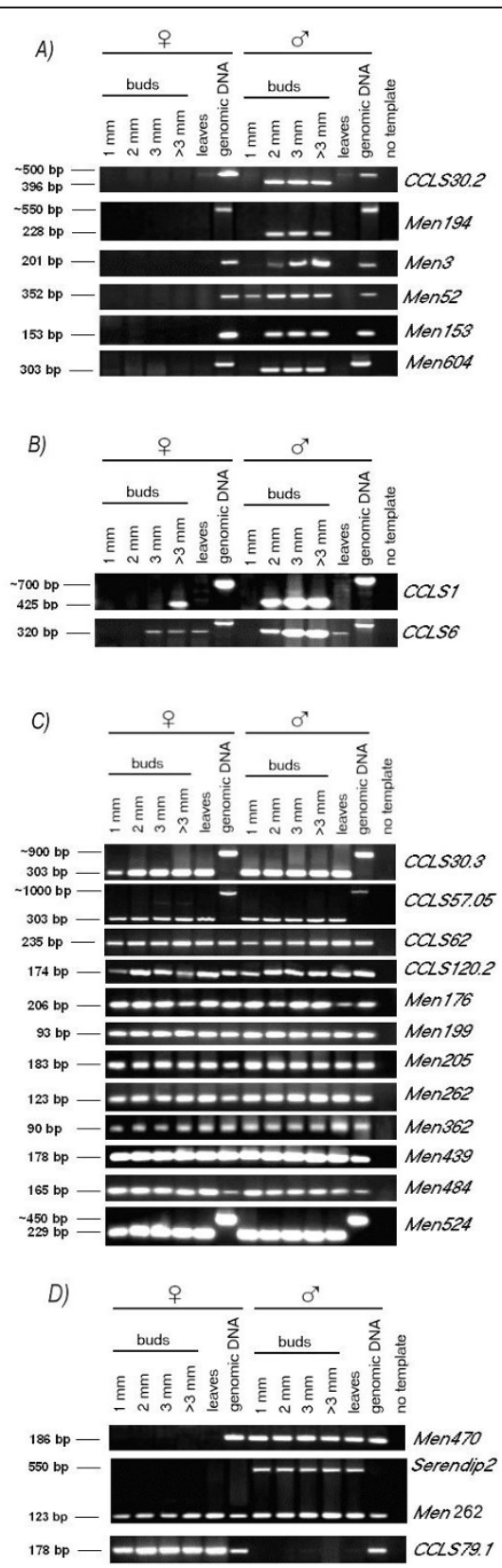

Figure 1 RT-PCR analyses of all the studied ESTs. Expression was investigated by RT-PCR analysis on the tissues indicated above each lane. The ESTs amplified are indicated on the right. Male buds of two $\mathrm{mm}$ length represent the stage when meiosis starts in anthers. Female meiosis starts in eight $\mathrm{mm}$ long female buds. Further details concerning the flower development in S. latifolia are summarized in Additional file 6: Supplementary table S4 (according to Farbos et al. [47]). (A) ESTs expressed exclusively in male flower buds. (B) ESTs starting to be expressed in male flower buds earlier than in female flower buds. (C) ESTs expressed in leaves and flower buds of both sexes. (D) ESTs showing sex specific expression in all the studied tissues. Two of them (Serendip2, and Men-470) are showing male specific expression while CCLS79.1 gene shows female specific expression. Men-262 is included to illustrate that Serendip2 is amplified with the same pair of primers and it serves also as a proof of the sufficient quality of templates. 
named it Serendip2 [GenBank: GU120088]. Serendip2 was expressed exclusively in male flower buds and leaves.

To elucidate the discrepancy between the expression patterns found here and those presented in previous research [22-24], we performed a homology search of the studied ESTs followed by a search for putative orthologous sequences in Arabidopsis thaliana (for the results, see Additional file 1: Supplementary table S1 and Additional file 3: Supplementary figures S1-11) and their expression patterns according to Genevestigator V3 [25,26] (for the results, see Additional file 1: Supplementary table S1; Genevestigator workspace file is included as Additional file 4). We found that the gene expression patterns described in A. thaliana were not in a contradiction to our results (cf. Additional file 1: Supplementary table S1 and the Figure 1).

In this article, we present sex-specific expression patterns of Men-470, Serendip2 and CCLS79.1; they are expressed in a sex-specific manner not only in flower buds, but also in the leaves of plants in the vegetative stage of development. At this stage, no expression of genes involved in the flower formation is present in $A$. thaliana (reviewed in [27]). Men-470 has already been studied using RT-PCR by other authors [24], who reported expression both in male and female flowers and in leaves (sex not specified). Using our primers, we observed transcription in males in all tissues studied, but no transcription in females. This result suggests that the expression of the copy amplified by our primer pair is already sex specific in the rosette stage. We can exclude the alternative possibility that our primers amplified only the Y-linked copy of Men- 470 by amplifying the sequence from females (XX). Serendip 2 is a new EST that was found in this study. Serendip 2 has an open reading frame along the whole sequence, but it has no homology to any known gene either at the DNA or protein level. The most interesting case is probably the CCLS79.1 gene. This sequence was amplified from genomic DNA samples of both females and males, suggesting that the difference in expression is not caused by sex linkage. The results we obtained in CCLS79.1 differ from the results obtained previously in Northern blots [22] that showed expression both in male and female flower buds and no expression in male leaves. The different results of our study can be explained by the fact that RT-PCR can selectively amplify one of several copies present in the genome.

Our results clearly show that male and female $S$. latifolia plants differ in the expression of at least three genes long before the initiation of flowering; this situation is analogous to the pregonadal stage in mammals [28]. Growth differences and sex specific expression are present in mammalian embryos in the preimplantation stage, long before the formation of sex organs. Similarly, sex-dimorphic gene expression has been found in gastrulating chicken embryos [29]. The main difference between plant and animal bodies is that plants do not possess a true germline and sexual organs develop relatively late in plant life. Given this pattern, our discovery of sexual dimorphism at the rosette stage is surprising. Indeed, contrary to animals, sexual dimorphism in plants at the vegetative stage before inflorescence initiation seems to be extremely rare. In classical dioecious model species, the earliest differences between male and female individuals are apparent in the inflorescence shape (in hop (Humulus) or hemp (Cannabis)). Spatial sex segregation, which is caused by differential seed germination and seedling survival (reviewed by [30]), is an indirect indication of the existence of sexual dimorphism in the vegetative stage of plants [31]. Additionally, the salt grass Distichlis spicata, a species characterized by spatial segregation of sexes, shows sex specific differences in susceptibility to colonization by a mycorrhizal fungus [30]. In this species, the sex specific differences even result in a strong inter-sexual competition [32]. As already listed in introduction, there are only a few indirect indications of the sexually dimorphic sex expression in the early vegetative state in S. latifolia that were obtained by previous studies [19-21]. The differences in expression patterns of three ESTs found in this study are the first qualitative differences between the sexes in the vegetative stage in S. latifolia. They are also the first described sequences in plants connected with the sexual dimorphism in the vegetative stage.

The existence of the sex specifically expressed genes in S. latifolia in the rosette stage suggests that there may be some, as yet undetected, physiological differences between sexes. We speculate that such hidden sexual dimorphism may be present in many dioecious species, and this study should inspire other scientists to test other dioecious species for sexual dimorphism in early vegetative stages. The existence of sexually dimorphic patterns means that the $S$. latifolia plants "know" their sex a long time before flowering, and this situation probably enables the plants to prepare for flowering in a sex specific manner.

\section{Conclusions}

To our knowledge, this study provides the first molecular evidence of early pre-flowering sexual dimorphism in angiosperms.

\section{Methods}

Orthology identification and search for the expression patterns in A. thaliana

Orthology data were obtained from OrthoMCL [33]. Files containing protein sequence data and orthology 
group information were downloaded from OrthoMCL version 4, currently containing genes from nine green plant genomes. The OrthoMCL clustering method provides a convenient, but necessarily imperfect means of estimating orthology and paralogy ([34], [35]). The highest BLAST hits can, in some cases, occur due to domain homologies, rather than homology to orthologs [36]. We performed phylogenetic analysis to avoid this misleading orthologue identification. Sequences for phylogenetic tree construction were obtained via a BLASTX homology search of the database of non-redundant protein sequences at NCBI (nr). All ESTs showing significant homology to known sequences were subjected to phylogenetic tree construction to further confirm their orthologues in other plant species. For the phylogenetic analysis, translated sequences were aligned using ClustalW version 1.83 [37], and the alignment was manually corrected using Seaview [38]. Ambiguously aligned parts of the sequences were excluded using Gblocks [39]. Phylogenetic trees were constructed by the maximum likelihood algorithm using PhyML version 3.0.1 [40] using the LG + Г4 + I model [41] and they were visualized using Dendroscope [42]. Branches were tested for reliability by approximate likelihood-ratio test [43]. Phylogenetic trees were rooted using an appropriate outgroup. Putative orthologues in A. thaliana were detected at all $A$. thaliana genes that grouped with a respective Silene latifolia EST with a high degree of confidence. The cut-off to detect orthology was set to 0.7. The expression patterns of the A. thaliana orthologues were searched using Genevestigator V3 $[25,26]$.

\section{Extraction of nucleic acids and RT-PCR}

S. latifolia plants were grown as described by Markova et al. [44]. Genomic DNA was isolated as described previously [45]. The sex of the plants was estimated at the rosette stage based on the length polymorphism between $\mathrm{X}$ and $\mathrm{Y}$ copy of the gene SlssX/Y (using the primers $\mathrm{c} 2 \mathrm{~B} 12+1$ and $\mathrm{c} 2 \mathrm{~B} 12-2)[46]$. RNA from a bulk sample of six male or female plants was isolated from rosette leaves (before the first flowering season; at the eight leaves stage) and flower buds of four different sizes (smaller than $1 \mathrm{~mm}$, between 1 and $2 \mathrm{~mm}$, between 2 and $3 \mathrm{~mm}$ and bigger than three $\mathrm{mm}$ ). For RNA isolation and reverse transcription, we used the same procedures as described previously [12]. To verify the results, RT-PCR was also performed on a single male and a single female with the same results as the bulk analysis. A list of all PCR primers and conditions used in this study is provided in Additional file 5: Supplementary Table S3. PCR products were analyzed on agarose gels and visualized under UV light in the presence of ethidium bromide. Expression was classified in a qualitative manner as present or absent.

\section{Additional material}

Additional file 1: Table S1. Complete list of the studied genes with available information concerning putative $A$. thaliana orthologues found in this study.

Additional file 2: Table S2: Comparison of the previously published results with the results obtained in this study The file compares the gene expression data obtained in this study with the previously published data. The published data on testing of $Y$-chromosome linkage are also summarized.

Additional file 3: Figures S1-11. The file contains these supplementary figures: Supplementary figure S1 - Phylogenetic analysis of the gene CCLS6. Supplementary figure S2 - Phylogenetic analysis of the gene CCLS30.2. Supplementary figure S3 - Phylogenetic analysis of the gene CCLS30.3. Supplementary figure S4 - Phylogenetic analysis of the gene CCLS57.05. Supplementary figure S5 - Phylogenetic analysis of the gene CCLS62. Supplementary figure S6 - Phylogenetic analysis of the gene CCLS120.2. Supplementary figure S7 - Phylogenetic analysis of the gene Men-194. Supplementary figure S8 - Phylogenetic analysis of the gene Men-439. Supplementary figure 59 - Phylogenetic analysis of the gene Men-484. Supplementary figure S10 - Phylogenetic analysis of the gene Men-524. Supplementary figure S11 - Phylogenetic analysis of the gene Men-604

Additional file 4: Genevestigator workspace file for all the found putative $\boldsymbol{A}$. thalina orthologs. This file contains expression data available for the found putative $A$. thaliana orthologs of the genes: CCLS6, CCLS30.2, CCLS30.3, CCLS57.05, CCLS62, CCLS120.2, Men-194, Men439, Men-484, Men-524 and Men-604. The file can be viewed using Genevestigator V3 at the URL: https://www.genevestigator.com/gv/user/ gvLogin.jsp (Registration is recommended.)

Additional file 5: Table S3. List of PCR primers and conditions Additional file 6: Supplementary Table S4: Flower development in Silene latifolia. The table compares development of male and female flowers in S. latifolia.

\section{Acknowledgements}

The authors thank Ms. Martina Kasikova and Magda Soukupova for technical assistance. This research was mainly supported by the project KJB600040801 (project of the Grant Agency of AS CR to J.Z.) and by the Institutional Research Plans (AV0Z50040507 and AV0Z50040702). This research was also supported by the project IAA600040801 to B.J. (Grant Agency of AS (R) and by project P501/10/0102 to B.V. (Grant Agency of the (zech Republic)

\section{Authors' contributions}

JZI conceived and designed the experiments. JZI, JZa and BJ performed the experiments. JZI analyzed the data. JZI, BJ and BV contributed reagents/ materials/analysis tools. BV discussed the paper. JZI and BJ wrote the paper. All authors read and approved the final manuscript.

Received: 17 December 2009 Accepted: 20 September 2010 Published: 20 September 2010

\section{References}

1. Williams TM, Carroll SB: Genetic and molecular insights into the development and evolution of sexual dimorphism. Nat Rev Genet 2009, 10:797-804.

2. Ober C, Loisel DA, Gilad Y: Sex-specific genetic architecture of human disease. Nat Rev Genet 2008, 9:911-922.

3. Dawson TE, Geber MA: Dimorphism in physiology and morphology. In Gender and Sexual Dimorphism in Flowering Plants. Edited by: Geber MA, Dawson TE, Delph LF. Berlin: Springer Verlag; 1999:175-215.

4. Ashman $\mathrm{TL}$ : The limits on sexual dimorphism in vegetative traits in a gynodioecious plant. Am Nat 2005, 166(Suppl):S5-S16.

5. Schulz A: Beiträge zur Kenntnis der Bestäubungseinrichtungen und Geschlechtsverteilung bei den Pflanzen. // Bibl Bot 1890, 17:182-196. 
6. Gehring JL: Temporal patterns in the development of sexual dimorphisms in Silene latifolia (Caryophyllaceae). Bull Torrey Bot Club 1993, 120:405-416.

7. Kaltz O, Shykoff JA: Male and female Silene latifolia plants differ in percontact risk of infection by a sexually transmitted desease. J Ecol 2001, 89:99-109

8. Delph LF, Frey FM, Steven JC, Gehring JL: Investigating the independent evolution of the size of floral organs via G-matrix estimation and artificial selection. Evol Dev 2004, 6:438-448.

9. Delph LF, Gehring JL, Arntz AM, Levri M, Frey FM: Genetic correlations with floral display lead to sexual dimorphism in the cost of reproduction. Am Nat 2005, 166(Suppl):S31-S41.

10. Farbos I, Veuskens J, Vyskot B, Oliveira M, Hinnisdaels S, Aghmir A, Mouras A, Negrutiu I: Sexual dimorphism in white campion: deletion on the $\mathrm{Y}$ chromosome results in a floral asexual phenotype. Genetics 1999, 151:1187-1196.

11. Matsunaga S, Uchida W, Kawano S: Sex-specific cell division during development of unisexual flowers in the dioecious plant Silene latifolia. Plant Cell Physiol 2004, 45:795-802.

12. Zluvova J, Nicolas M, Berger A, Negrutiu I, Moneger F: Premature arrest of the male flower meristem precedes sexual dimorphism in the dioecious plant Silene latifolia. Proc Natl Acad Sci USA 2006, 103:18854-18859.

13. Kazama Y, Fujiwara MT, Koizumi A, Nishihara K, Nishiyama R, Kifune E, Abe T, Kawano S: A SUPERMAN-like gene is exclusively expressed in female flowers of the dioecious plant Silene latifolia. Plant Cell Physiol 2009, 50:1127-1141.

14. Bateman AJ: Intrasexual selection in Drosophila. Heredity 2:349-368.

15. Hall DW, Kirkpatrick M, West B: Runaway sexual selection when female preferences are directly selected. Evolution 2000, 54:1862-1869.

16. Waelti MO, Page PA, Widmer A, Schiestl FP: How to be an attractive male: floral dimorphism and attractiveness to pollinators in a dioecious plant. BMC Evol Biol 2009, 9:190.

17. Laporte MM, Delph LF: Sex-specific physiology and source-sink relations in the dioecious plant Silene latifolia. Oecologia 1996, 106:63-72.

18. Steven JC, Delph LF, Brodie ED III: Sexual dimorphism in the quantitativegenetic architecture of floral, leaf, and allocation traits in Silene latifolia. Evolution 2007, 61:42-57.

19. Purrington $C B$, Schmitt J: Sexual dimorphism of dormancy and survivorship in buried seeds of Silene latifolia. Journal of Ecology 1995, 83:795-800.

20. Purrington $C B$, Schmitt J: Consequences of sexually dimorphic timing of emergence and flowering in Silene latifolia. J Ecol 1998, 86:286-393.

21. Doust JL, O'Brien G, Doust LL: Effect of Density on Secondary Sex Characteristics and Sex Ratio in Silene alba (Caryophyllaceae). American Journal of Botany 1987, 74:40-46.

22. Barbacar N, Hinnisdaels S, Farbos I, Moneger F, Lardon A, Delichere C, Mouras A, Negrutiu I: Isolation of early genes expressed in reproductive organs of the dioecious white campion (Silene latifolia) by subtraction cloning using an asexual mutant. Plant J 1997, 12:805-817.

23. Scutt CP, Li T, Robertson SE, Willis ME, Gilmartin PM: Sex determination in dioecious Silene latifolia. Effects of the $Y$ chromosome and the parasitic smut fungus (Ustilago violacea) on gene expression during flower development. Plant Physiol 1997, 114:969-979.

24. Scutt $C P$, Jenkins T, Furuya M, Gilmartin PM: Male specific genes from dioecious white campion identified by fluorescent differential display. Plant Cell Physiol 2002, 43:563-57232.

25. Zimmermann P, Hirsch-Hoffmann M, Hennig L, Gruissem W: GENEVESTIGATOR. Arabidopsis microarray database and analysis toolbox. Plant Physiol 2004, 136:2621-2632.

26. Hruz T, Laule O, Szabo G, Wessendorp F, Bleuler S, Oertle L, Widmayer P Gruissem W, Zimmermann P: Genevestigator v3: a reference expression database for the meta-analysis of transcriptomes. Adv Bioinformatics 2008 2008:420747.

27. Krizek BA, Fletcher JC: Molecular mechanisms of flower development: an armchair guide. Nat Rev Genet 2005, 6:688-698.

28. Yadav BR, King WA, Betteridge KJ: Relationships between the completion of first cleavage and the chromosomal complement, sex, and developmental rates of bovine embryos generated in vitro. Mol Reprod Dev 1993, 36:434-439.
29. Zhang SO, Mathur S, Hattem G, Tassy O, Pourquié O: Sex-dimorphic gene expression and ineffective dosage compensation of Z-linked genes in gastrulating chicken embryos. BMC Genomics 2010, 11:13.

30. Eppley SM, Mercer CA, Haaning C, Graves CB: Sex-specific variation in the interaction between Distichlis spicata (Poaceae) and mycorrhizal fungi. Am J Bot 2009, 96:1967-1973.

31. Bierzychudek $P$, Eckhart V: Spatial segregation of sexes of dioecious plants. Am Nat 1988, 132.

32. Mercer CA, Eppley SM: Inter-sexual competition in a dioecious grass Oecologia 2010.

33. Chen F, Mackey AJ, Stoeckert CJ Jr, Roos DS: OrthoMCL-DB: querying a comprehensive multi-species collection of ortholog groups. Nucleic Acids Res 2006, , 34 Database: D363-368.

34. Li L, Stoeckert CJ Jr, Roos DS: OrthoMCL: identification of ortholog groups for eukaryotic genomes. Genome Res 2003, 13:2178-2189.

35. Chen F, Mackey AJ, Vermunt K, Roos DS: Assessing Performance of Orthology Detection Strategies Applied to Eukaryotic Genomes. PLOS ONE 2007, 2:e383.

36. Lindqvist C, Scheen AC, Yoo MJ, Grey P, Oppenheimer DG, LeebensMack JH, Soltis DE, Soltis PS, Albert VA: An expressed sequencetag (EST) library from developing fruits of an Hawaiian endemic mint (Stenogyne rugosa, Lamiaceae): characterization and microsatellite markers. BMC Plant Biol 2006, 6:16.

37. Thompson JD, Higgins DG, Gibson TJ: CLUSTAL W: improving the sensitivity of progressive multiple sequence alignment through sequence weighting, position-specific gap penalties and weight matrix choice. Nucleic Acids Res 1994, 22:4673-4680.

38. Galtier N, Gouy M, Gautier C: SEAVIEW and PHYLO_WIN: two graphic tools for sequence alignment and molecular phylogeny. Comput Appl Biosci 12:543-548.

39. Castresana J: Selection of conserved blocks from multiple alignments for their use in phylogenetic analysis. Mol Biol Evol 2000, 17:540-552.

40. Guindon S, Gascuel O: A simple, fast, and accurate algorithm to estimate large phylogenies by maximum likelihood. Syst Biol 2003, 52:696-704.

41. Le SQ, Gascuel O: An improved general amino acid replacement matrix. Mol BiolEvol 2008, 25:1307-1320.

42. Huson D, Richter D, Rausch C, Dezulian T, Franz M, Rupp R: Dendroscope: An interactive viewer for large phylogenetic trees. BMC Bioinformatics 2007, 8:460.

43. Anisimova M, Gascuel O: Approximate likelihood-ratio test for branches: A fast, accurate, and powerful alternative. Syst Biol 2006, 55:539-552.

44. Markova M, Michu E, Vyskot B, Janousek B, Zluvova J: An interspecific hybrid as a tool to study phylogenetic relationships in plants using the GISH technique. Chromosome Res 2007, 15:1051-1059.

45. Zluvova J, Lengerova $M$, Markova $M$, Hobza $R$, Nicolas $M$, Vyskot $B$ Charlesworth D, Negrutiu I, Janousek B: The inter-specific hybrid Silene latifolia $\times$ S. viscosa reveals early events of sex chromosome evolution. Evol Dev 2005, 7:327-336

46. Filatov DA: Evolutionary history of Silene latifolia sex chromosomes revealed by genetic mapping of four genes. Genetics 2005, 170:975-979.

47. Farbos I, Oliveira M, Negrutiu I, Mouras A: Sex organ determination and differentiation in the dioecious plant Melandrium album (Silene latifolia): cytological and histological analysis. Sex Plant Reprod 1997, 10:155-167.

doi:10.1186/1471-2229-10-208

Cite this article as: Zluvova et al:: Dioecious Silene latifolia plants show sexual dimorphism in the vegetative stage. BMC Plant Biology 2010 10:208. 